\title{
Design of Space-Curve Meshing-Wheels with Unequal Tine Radii
}

\author{
Jiang Ding - Yangzhi Chen* -Yueling Lv \\ South China University of Technology, China
}

In some recent papers a new gear mechanism named Space-Curve Meshing-Wheel (SCMW) has been proposed by the present authors. However, the research presented was limited in the equal tine radius case. This paper presents a method to solve the SCMW design with unequal tine radii. As a consequence, with a pair of contact curves, the tine radii of the driving and driven wheels can be selected independently according to actual need. A design example is illustrated in detail and testified in both simulation and practical experiment. This method provides a design fundamental for the SCMWs to optimize the tine shapes according to strength conditions, and therefore it can extend their application in industrial.

Keywords: gear, Space-Curve Meshing-Wheel, unequal tine radius, contact curve

\section{INTRODUCTION}

A gear mechanism named Space-Curve MeshingWheel (SCMW) was invented by Chen et al. [1] to [3]. The SCMW is based on the theory of space curve meshing instead of traditional space surface meshing [4] to [8]. Space curve meshing is a transmission through continuous point contacts between two conjugate curves. With a large transmission ratio, small size and light weight, SCMW is highly convenient to be applied in the transmission between two intersecting axes in the small space inside micro machines. After recent research on space curve meshing equations [1] to [3], contact ratio [9], design criterion [10] and manufacture technology [11], the SCMW has possessed an integrated application like micro reducers [12].

However, the research published was limited in the equal tine radius case. Furthermore, the space curve meshing equations obtained at the meshing point were relevant to the tine radii, and therefore the wheel pair of the SCMW must be in a one-toone correspondence. But in practical application, the demanding tine radii of the driving and driven wheels are not always the same. The tine radii depend on the demanding strength corresponding to their working conditions. If the tine radii of the driving and driven wheels are irrelevant from each other, they can be designed independently.

This paper presents a design method to make the tines of SCMW irrelevant in the unequal tine radius case. To testify the method, an example is designed, and both simulation and practical experiment are carried out with the same data in the example.

\section{DESIGN FUNDAMENTAL}

The essence of the space-curve meshing is transmission through continuous point contacts between two conjugate curves [1]. To provide contact curves smooth and slight objectives like tines, for instance, should be designed.

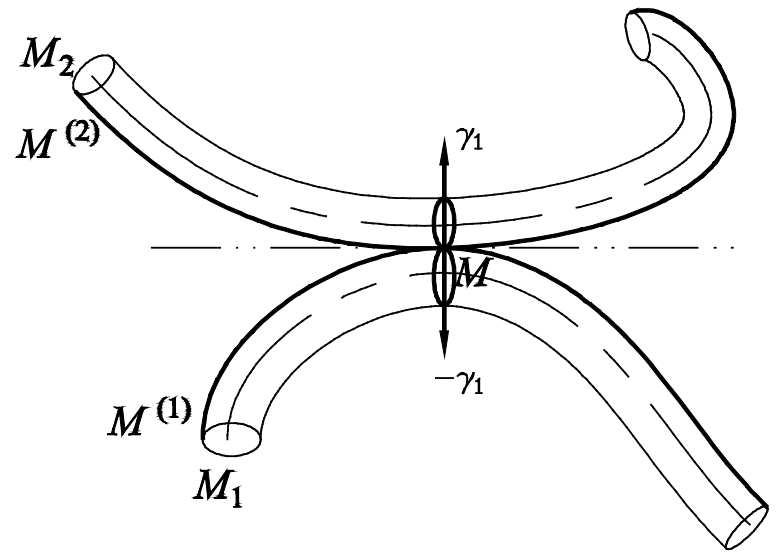

Fig. 1. Cylindrical tines and contact curves

For reasons of simplicity, cylindrical tines have been adopted in this paper as in previous papers [2] to [3]. As shown in Fig. 1, two invariant circles are tangent at the meshing point each moment. The circle centers are along the contact vector direction and at the opposite sides of the meshing point (indicated as $-\gamma_{1}$ and $\gamma_{1}$ in Fig. 1). After the curves finish the whole meshing, point after point, the two circles form the cylindrical shapes of the driving and driven tines, respectively. In Fig. 1, $M$ is the meshing point at each moment, while $M^{(1)}$ and $M^{(2)}$ are the corresponding points at the driving and driven tines, respectively. The point set of $M^{(1)}$ is the driving contact curve; the point set of $M^{(2)}$ is the driven contact curve.

According to the design depicted above, for the same contact curves, the driving and driven tines can adopt circles with different radii, attaining the cylindrical tines with different radii. Even the tines with variable radii can be designed in this way. As the 
circles are always in opposite directions at the meshing point, the driving and driven tines will be irrelevant from each other. In theory, the radii of the tines are free to choose as long as there is enough room. In practical application, the tine radii are determined according to the strength conditions.

\section{DESIGN METHOD}

\subsection{Space Meshing Coordinates}

The equations of the contact curves and the center curves are all calculated in the space meshing coordinates, which are established as Fig. 2.

The fixed coordinates for the driving and driven wheels are given as $o-x y z$ and $o_{p}-x_{p} y_{p} z_{p}$, respectively. Planes $x_{p} o_{p} z_{p}$ and $x o z$ are in the same plane. Denote the distance from point $o_{p}$ to axis $z$ as $a$ and the distance to axis $x$ as $b$. Included angle between axis $z$ and axis $z_{p}$ is $(\pi-\theta)$, where $\theta$ is the included angle between the angular velocity vectors of the driving and driven wheels.

$o_{1}-x_{1} y_{1} z_{1}$ and $o_{2}-x_{2} y_{2} z_{2}$ are relatively static with the driving and driven wheels. They are rotating coordinates with respect to $o-x y z$ and $o_{p}-x_{p} y_{p} z_{p}$, respectively. At the initial moment, $o_{1}-x_{1} y_{1} z_{1}$ coincides with $o-x y z$, while $o_{2}-x_{2} y_{2} z_{2}$ coincides with $o_{p}-x_{p} y_{p} z_{p}$. At any moment, point $o_{1}$ coincides with point $o$, and axis $z_{1}$ coincides with axis $z$; point $O_{2}$ coincides with point $o_{p}$, and axis $z_{2}$ coincides with axis $z_{p}$. After meshing begins, $o_{1}-x_{1} y_{1} z_{1}$ rotates around axis $z_{1}$, while $o_{2}-x_{2} y_{2} z_{2}$ rotates around axis $z_{2}$.

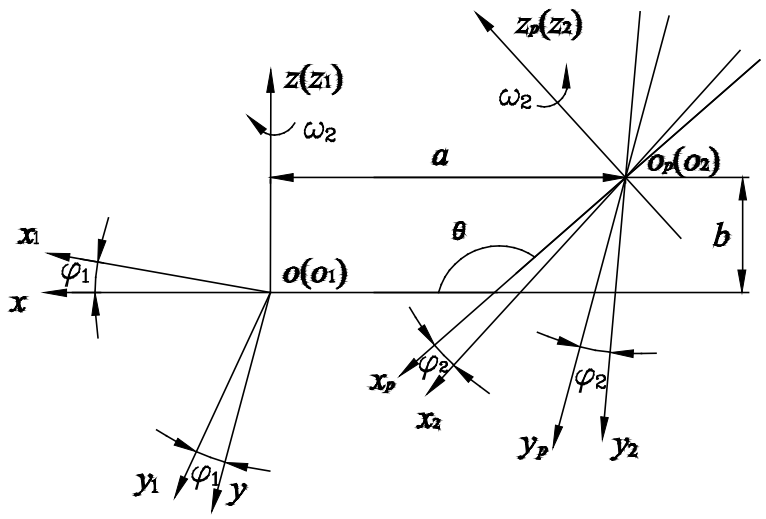

Fig. 2. Space meshing coordinates

The transformation matrix from $o_{1}-x_{1} y_{1} z_{1}$ to $o-x y z$ is as Eq. (1) [13].

$$
M_{o 1}=\left[\begin{array}{cccc}
\cos \varphi_{1} & \sin \varphi_{1} & 0 & 0 \\
-\sin \varphi_{1} & \cos \varphi_{1} & 0 & 0 \\
0 & 0 & 1 & 0 \\
0 & 0 & 0 & 1
\end{array}\right] .
$$

The transformation matrix from $o_{1}-x_{1} y_{1} z_{1}$ to $o_{2}-x_{2} y_{2} z_{2}$ is as Eq. (2) [13].

$$
\begin{aligned}
& M_{21}=\left[\begin{array}{c}
-\cos \varphi_{1} \cos \varphi_{2} \cos \theta-\sin \varphi_{1} \sin \varphi_{2} \\
\cos \varphi_{1} \sin \varphi_{2} \cos \theta-\sin \varphi_{1} \cos \varphi_{2} \\
\cos \varphi_{1} \sin \theta \\
0
\end{array}\right. \\
& -\sin \varphi_{1} \cos \varphi_{2} \cos \theta+\cos \varphi_{1} \sin \varphi_{2} \\
& \sin \varphi_{1} \sin \varphi_{2} \cos \theta+\cos \varphi_{1} \cos \varphi_{2} \\
& \sin \varphi_{1} \sin \theta \\
& 0 \\
& \left.\begin{array}{cc}
-\cos \varphi_{2} \sin \theta & -a \cos \varphi_{2} \cos \theta+b \cos \varphi_{2} \sin \theta \\
\sin \varphi_{2} \sin \theta & a \sin \varphi_{2} \cos \theta-b \sin \varphi_{2} \sin \theta \\
-\cos \theta & a \sin \theta+b \cos \theta \\
0 & 1
\end{array}\right] .
\end{aligned}
$$

As shown in Fig. 2, suppose: $\varpi_{1}$ and $\varpi_{2}$ are the angular velocities of the driving and driven wheels; $\varphi_{1}$ and $\varphi_{2}$ are the rotation angles of the driving and driven wheels after the meshing begins; $i_{12}$ is the transmission ratio. The kinematic relations are obtained as Eqs. (3) and (4):

$$
\begin{aligned}
& \varpi_{1}=i_{12} \varpi_{2}, \\
& \varphi_{1}=i_{12} \varphi_{2} .
\end{aligned}
$$

\subsection{Kinematical Equation}

According to [1], the motion at the meshing point should satisfy the kinematical equation as Eq. (5):

$$
\boldsymbol{v}_{12} \cdot \boldsymbol{\beta}=0,
$$

where $\boldsymbol{v}_{12}$ is the relative velocity at the meshing point between the driving and driven tines, and $\boldsymbol{\beta}=\left[\begin{array}{c}\beta_{x} \\ \beta_{y} \\ \beta_{z}\end{array}\right]$ is the unit normal vector of the driving contact curve in $o-x y z$.

Suppose that $\boldsymbol{\beta}(1)$ is the unit normal vector of the driving curve in $o_{1}-x_{1} y_{1} z_{1}$. Considering Eq. (1), we get the equation of the unit normal vector as Eq. (6): 


$$
\boldsymbol{\beta}=\left[\begin{array}{c}
\beta_{x}^{(1)} \cos \varphi_{1}+\beta_{y}^{(1)} \sin \varphi_{1} \\
-\beta_{x}^{(1)} \sin \varphi_{1}+\beta_{y}^{(1)} \cos \varphi_{1} \\
\beta_{z}^{(1)}
\end{array}\right]
$$

The velocities at the meshing point of the driving and driven tines are as Eqs. (7) and (8):

$$
\begin{aligned}
& \boldsymbol{v}_{1}=\left[\begin{array}{c}
y_{M} \varpi_{1} \\
-x_{M} \varpi_{1} \\
0
\end{array}\right], \\
& \boldsymbol{v}_{2}=\left[\begin{array}{c}
y_{M} \varpi_{2} \cos \theta \\
-\left(z_{M}-b\right) \varpi_{2} \sin \theta-\left(x_{M}+a\right) \varpi_{2} \cos \theta \\
y_{M} \varpi_{2} \sin \theta
\end{array}\right] \text {. } \\
& \left(y_{M}^{(1)} \beta_{x}^{(1)}-x_{M}^{(1)} \beta_{y}^{(1)}\right)\left(\varpi_{1}-\varpi_{2} \cos \theta\right)+ \\
& +a \varpi_{2} \cos \theta\left(-\beta_{x}^{\left({ }^{(1)}\right.} \sin \varphi_{1}+\beta_{y}^{(1)} \cos \varphi_{1}\right)+ \\
& +\left(z_{M}^{(1)}-b\right) \varpi_{2} \sin \theta\left(\beta_{y}^{(1)} \cos \varphi_{1}-\beta_{x}^{(1)} \sin \varphi_{1}\right)+ \\
& +\left(x_{M}^{(1)} \sin \varphi_{1}-y_{M}^{(1)} \cos \varphi_{1}\right) \varpi_{2} \sin \theta\left(\beta_{z}^{(1)}\right)=0 .
\end{aligned}
$$
Eq. (9):

The relative velocity at the meshing point is as

$$
v_{12}=v_{1}-v_{2}
$$

Considering Eq. (1), we get:

$$
\left\{\begin{array}{l}
x_{M}=x_{M}^{(1)} \cos \varphi_{1}+y_{M}^{(1)} \sin \varphi_{1} \\
y_{M}=-x_{M}^{(1)} \sin \varphi_{1}+y_{M}^{(1)} \cos \varphi_{1} \\
z_{M}=z_{M}^{(1)}
\end{array}\right.
$$

Substituting Eqs. (7), (8) and (10) into Eq. (9), relative velocity at the meshing point as Eq. (11) is obtained:

$$
\boldsymbol{v}_{12}=\left[\begin{array}{c}
\left(-x_{M}^{(1)} \sin \varphi_{1}+y_{M}^{(1)} \cos \varphi_{1}\right)\left(\varpi_{1}-\varpi_{2} \cos \theta\right) \\
-\left(x_{M}^{(1)} \cos \varphi_{1}+y_{M}^{(1)} \sin \varphi_{1}\right) \varpi_{1}+\left(z_{M}^{(1)}-b\right) \varpi_{2} \sin \theta+\left(x_{M}^{(1)} \cos \varphi_{1}+y_{M}^{(1)} \sin \varphi_{1}+a\right) \varpi_{2} \cos \theta \\
-\left(-x_{M}^{(1)} \sin \varphi_{1}+y_{M}^{(1)} \cos \varphi_{1}\right) \varpi_{2} \sin \theta
\end{array}\right]
$$

From Eq. (12) above, the relationship between $\varphi_{1}$ and $t$ can be gained, and then the equations of the contact curves and the center curves can be derived. As neither $r_{1}$ nor $r_{2}$ exists in Eq. (12), we can conclude that the kinematical equation is only related to the properties of the driving contact curve $\left(x_{M}^{(1)}\right.$, $y_{M}^{(1)}, z_{M}^{(1)}, \beta_{x}^{(1)}, \beta_{y}^{(1)}$ and $\left.\beta_{z}^{(1)}\right)$ and the kinematic relations $\left(a, b, \theta, \varpi_{1}, \varpi_{2}\right.$, and $\left.\varphi_{1}\right)$ between the driving and driven contact curves, but not the tine radii.

\subsection{Equations of Contact Curves}

Denote the matrices of $M^{(1)}$ in $o_{1}-x_{1} y_{1} z_{1}$ and $M^{(2)}$ in $o_{2}-x_{2} y_{2} z_{2}$ as $\left[\begin{array}{c}x_{M}^{(1)} \\ y_{M}^{(1)} \\ z_{M}^{(1)}\end{array}\right]$ and $\left[\begin{array}{c}x_{M}^{(2)} \\ y_{M}^{(2)} \\ z_{M}^{(2)}\end{array}\right]$, respectively. The equation of the driving contact curve is given as Eq. (13):

$$
\left\{\begin{array}{l}
x_{M}^{(1)}=x_{M}^{(1)}(t) \\
y_{M}^{(1)}=y_{M}^{(1)}(t) . \\
z_{M}^{(1)}=z_{M}^{(1)}(t)
\end{array}\right.
$$

Considering Eqs. (2) and (13), the equation of the driven contact curve as Eq. (14) can be obtained:

$$
\left\{\begin{aligned}
x_{M}^{(2)}= & \left(-\cos \varphi_{1} \cos \varphi_{2} \cos \theta-\sin \varphi_{1} \sin \varphi_{2}\right) x_{M}^{(1)}+ \\
& +\left(-\sin \varphi_{1} \cos \varphi_{2} \cos \theta+\cos \varphi_{1} \sin \varphi_{2}\right) y_{M}^{(1)}- \\
& -\cos \varphi_{2} \sin \theta z_{M}^{(1)}-a \cos \varphi_{2} \cos \theta+b \cos \varphi_{2} \sin \theta \\
y_{M}^{(2)}= & \left(\cos \varphi_{1} \sin \varphi_{2} \cos \theta-\sin \varphi_{1} \cos \varphi_{2}\right) x_{M}^{(1)}+ \\
& +\left(\sin \varphi_{1} \sin \varphi_{2} \cos \theta+\cos \varphi_{1} \cos \varphi_{2}\right) y_{M}^{(1)}+ \\
& +\sin \varphi_{2} \sin \theta z_{M}^{(1)}+a \sin \varphi_{2} \cos \theta-b \sin \varphi_{2} \sin \theta \\
z_{M}^{(2)}= & \cos \varphi_{1} \sin \theta x_{M}^{(1)}+\sin \varphi_{1} \sin \theta y_{M}^{(1)}-\cos \theta z_{M}^{(1)}+ \\
& +a \sin \theta+b \cos \theta
\end{aligned}\right.
$$




\subsection{Equations of Center Curves}

Denote $\gamma$ and $\gamma^{(1)}$ as unit binormal vectors of the driving curve in $o-x y z$ and $o_{1}-x_{1} y_{1} z_{1}$, respectively. As shown in Figs. 1 and 3, at every meshing moment, if each meshing point $M(t)$ moves a distance of $r_{1}$ in the direction of $-\gamma^{(1)}$, the corresponding point in the driving center curve is obtained; if each meshing point $M(t)$ moves a distance of $r_{2}$ in the direction of $\gamma(t)$, the corresponding point in the driven center curve is obtained. The tine radii as Eqs. (15) and (16) can be derived:

$$
\begin{aligned}
& \boldsymbol{r}_{1}(t)=-r_{1} \gamma(t)=\overrightarrow{M M_{1}}, \\
& \boldsymbol{r}_{2}(t)=r_{2} \gamma(t)=\overrightarrow{M M_{2}} .
\end{aligned}
$$

Denote $M_{1}$ and $\overrightarrow{M M_{1}}$ in $o_{1}-x_{1} y_{1} z_{1}$ as $\left[\begin{array}{c}x_{M_{1}}^{(1)} \\ y_{M_{1}}^{(1)} \\ z_{M_{1}}^{(1)}\end{array}\right]$ and $\left[\begin{array}{l}x_{M M_{1}}^{(1)} \\ y_{M M_{1}}^{(1)} \\ z_{M M_{1}}^{(1)}\end{array}\right], M_{2}$ and $\overrightarrow{M M_{2}}$ in $o_{2}-x_{2} y_{2} z_{2}$, as $\left[\begin{array}{l}x_{M_{1}}^{(2)} \\ y_{M_{1}}^{(2)} \\ z_{M_{1}}^{(2)}\end{array}\right]$ and $\left[\begin{array}{l}x_{M M_{2}}^{(2)} \\ y_{M M_{2}}^{(2)} \\ z_{M M_{2}}^{(2)}\end{array}\right]$, respectively.

The vector addition triangles are shown in Fig. 3. From Fig. 3, Eqs. (17) and (18) can be derived.

$$
\begin{aligned}
& {\left[\begin{array}{l}
x_{M_{1}}^{(1)} \\
y_{M_{1}}^{(1)} \\
z_{M_{1}}^{(1)}
\end{array}\right]=\left[\begin{array}{l}
x_{M}^{(1)} \\
y_{M}^{(1)} \\
z_{M}^{(1)}
\end{array}\right]+\left[\begin{array}{l}
x_{M M_{1}}^{(1)} \\
y_{M M_{1}}^{(1)} \\
z_{M M_{1}}^{(1)}
\end{array}\right],} \\
& {\left[\begin{array}{l}
x_{M_{2}}^{(2)} \\
y_{M_{2}}^{(2)} \\
z_{M_{2}}^{(2)}
\end{array}\right]=\left[\begin{array}{l}
x_{M}^{(2)} \\
y_{M}^{(2)} \\
z_{M}^{(2)}
\end{array}\right]+\left[\begin{array}{l}
x_{M M_{2}}^{(2)} \\
y_{M M_{2}}^{(2)} \\
z_{M M_{2}}^{(2)}
\end{array}\right] .}
\end{aligned}
$$

According to Eq. (17), the equation of the driving center curves is as Eq. (19):

$$
\left\{\begin{array}{l}
x_{M_{1}}^{(1)}=x_{M}^{(1)}+x_{M M_{1}}^{(1)} \\
y_{M_{1}}^{(1)}=y_{M}^{(1)}+y_{M M_{1}}^{(1)} \\
z_{M_{1}}^{(1)}=z_{M}^{(1)}+z_{M M_{1}}^{(1)}
\end{array}\right.
$$

According to Eqs. (2) and (18), the equation of the driven center curves is as Eq.(20):

$$
\left\{\begin{aligned}
x_{M_{2}}^{(2)} & \left(-\cos \varphi_{1} \cos \varphi_{2} \cos \theta-\sin \varphi_{1} \sin \varphi_{2}\right)\left(x_{M}^{(1)}+x_{M M_{2}}^{(1)}\right)+ \\
& +\left(-\sin \varphi_{1} \cos \varphi_{2} \cos \theta+\cos \varphi_{1} \sin \varphi_{2}\right)\left(y_{M}^{(1)}+y_{M M_{2}}^{(1)}\right)- \\
& -\cos \varphi_{2} \sin \theta\left(z_{M}^{(1)}+z_{M M_{2}}^{(1)}\right)+ \\
& +\left(-a \cos \varphi_{2} \cos \theta+b \cos \varphi_{2} \sin \theta\right) \\
y_{M_{2}}^{(2)} & \left(\cos \varphi_{1} \sin \varphi_{2} \cos \theta-\sin \varphi_{1} \cos \varphi_{2}\right)\left(x_{M}^{(1)}+x_{M M_{2}}^{(1)}\right)+ \\
& +\left(\sin \varphi_{1} \sin \varphi_{2} \cos \theta+\cos \varphi_{1} \cos \varphi_{2}\right)\left(y_{M}^{(1)}+y_{M M_{2}}^{(1)}\right)+ \\
& +\sin \varphi_{2} \sin \theta\left(z_{M}^{(1)}+z_{M M_{2}}^{(1)}\right)+ \\
& +\left(a \sin \varphi_{2} \cos \theta-b \sin \varphi_{2} \sin \theta\right) \\
z_{M_{2}}^{(2)} & =\cos \varphi_{1} \sin \theta\left(x_{M}^{(1)}+x_{M M_{2}}^{(1)}\right)+ \\
& +\sin \varphi_{1} \sin \theta\left(y_{M}^{(1)}+y_{M M_{2}}^{(1)}\right)- \\
& -\cos \theta\left(z_{M}^{(1)}+z_{M M_{2}}^{(1)}\right)+(a \sin \theta+b \cos \theta)
\end{aligned}\right.
$$

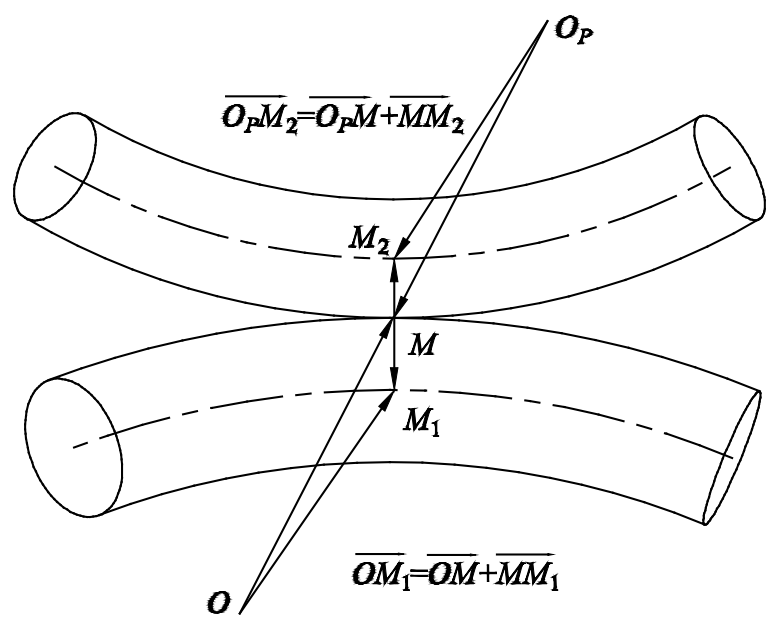

Fig. 3. Vector addition triangles

\section{DESIGN EXAMPLE}

\subsection{Equation of Driving Contact Curve}

In the reference [1] to [3], a helix curve was given as an example. In comparison, the same curve is adopted as the driving contact curve, as shown in Fig. 4a. The Equation of $M^{(1)}$ in $o_{1}-x_{1} y_{1} z_{1}$ is as Eq. (21):

$$
\left\{\begin{array}{l}
x_{M}^{(1)}=m \cos t \\
y_{M}^{(1)}=m \sin t \\
z_{M}^{(1)}=n \pi+n t
\end{array} \quad\left(-\pi \leq t \leq-\frac{\pi}{2}\right),\right.
$$

where $m$ is the helix radius of the driving curve; $n$ is the pitch parameter of the driving curve, denoting the 
pitch as $p, n=p / 2 \pi ; t$ is the parameter indicating the scope of the helix curve. $-\pi \leq t \leq-\pi / 2$ means a quarter circle of the helix curve. When $t=-\pi$, the driving and driven tines begin to mesh; when $t=-\pi / 2$, the two tines begin to separate. The lengths of the driving and driven contact curves are directly controlled by the scope of $t$ as needed.

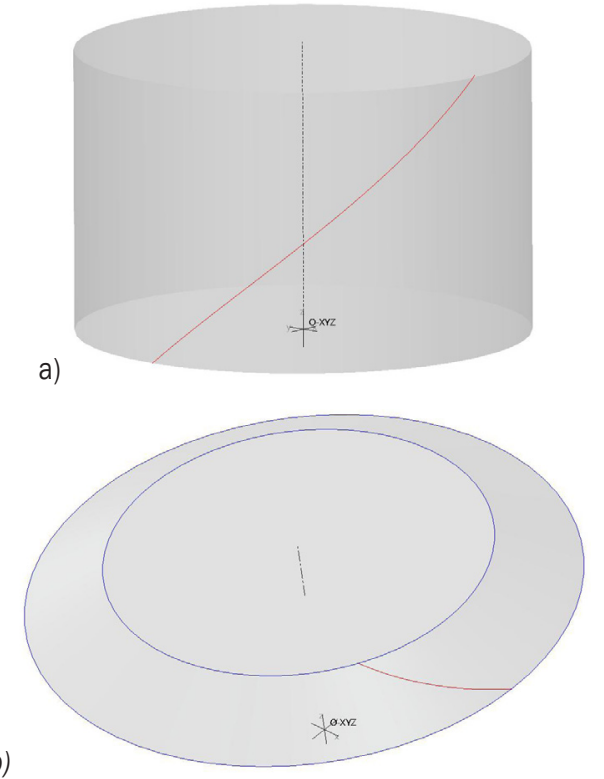

Fig. 4. a) Driving and b) driven contact curves

The unit normal vector and the unit binormal vector of the driving curve are derived as Eqs. (22) and (23).

$$
\begin{gathered}
\beta^{(1)}=\left[\begin{array}{l}
\beta_{x}^{(1)} \\
\beta_{y}^{(1)} \\
\beta_{z}^{(1)}
\end{array}\right]=\left[\begin{array}{c}
-\cos t \\
-\sin t \\
0
\end{array}\right], \\
\gamma^{(1)}=\left[\begin{array}{l}
\gamma_{x}^{(1)} \\
\gamma_{y}^{(1)} \\
\gamma_{z}^{(1)}
\end{array}\right]=\left[\begin{array}{c}
\frac{n \sin t}{\sqrt{n^{2}+m^{2}}} \\
-\frac{n \cos t}{\sqrt{n^{2}+m^{2}}} \\
\frac{m}{\sqrt{n^{2}+m^{2}}}
\end{array}\right] .
\end{gathered}
$$

\subsection{Kinematic Equation}

Substituting Eqs. (21) and (22) into Eq. (12), the kinematic equation as Eq. (24) is obtained :

$$
\varpi_{2} \sin \left(\varphi_{1}-t\right)[a \cos \theta+(n \pi+n t-b) \sin \theta]=0 .
$$

Considering $-\pi \leq t \leq-\pi / 2$ and $0<\varphi_{1}$, the relationship between $\varphi_{1}$ and $t$ can be deviced:

$$
\varphi_{1}=t+\pi \text {. }
$$

\subsection{Equation of Driven Contact Curve}

According to Eqs. (4), (14), (21) and (25), the equation of $M^{(2)}$ in $o_{2}-x_{2} y_{2} z_{2}$ is as Eq. (26):

$$
\left\{\begin{array}{c}
x_{M}^{(2)}=[(m-a) \cos \theta-(n \pi+n t-b) \sin \theta] \cdot \cos \frac{t+\pi}{i_{12}} \\
y_{M}^{(2)}=-[(m-a) \cos \theta-(n \pi+n t-b) \sin \theta] \cdot \sin \frac{t+\pi}{i_{12}} . \\
z_{M}^{(2)}=-(m-a) \sin \theta-(n \pi+n t-b) \cos \theta
\end{array}\right.
$$

Obviously, Eq. (26) is a conical helix curve, as shown in Fig. 4b.

\subsection{Equations of Center Curves}

According to Eqs. (15) and (16), the radii of the driving and driven tines are as Eqs. (27) and (28) in $o_{1}-x_{1} y_{1} z_{1}$.

$$
\begin{aligned}
& {\left[\begin{array}{l}
x_{M M_{1}}^{(1)} \\
y_{M M_{1}}^{(1)} \\
z_{M M_{1}}^{(1)}
\end{array}\right]=-r_{1} \gamma^{(1)},} \\
& {\left[\begin{array}{l}
x_{M M_{2}}^{(1)} \\
y_{M M_{2}}^{(1)} \\
z_{M M_{2}}^{(1)}
\end{array}\right]=r_{2} \gamma^{(1)} .}
\end{aligned}
$$

From Eqs. (19), (21), (23) and (27), the equation of the driving center curve is derived as Eq. (29):

$$
\left\{\begin{array}{l}
x_{M_{1}}^{(1)}=m \cos t-\frac{r_{1} n \sin t}{\sqrt{n^{2}+m^{2}}} \\
y_{M_{1}}^{(1)}=m \sin t+\frac{r_{1} n \cos t}{\sqrt{n^{2}+m^{2}}} . \\
z_{M_{1}}^{(1)}=n \pi+n t-\frac{r_{1} m}{\sqrt{n^{2}+m^{2}}}
\end{array}\right.
$$

From Eqs. (4), (20), (21), (23), (25) and (28), the equation of the driven center curve is derived as Eq. (30): 


$$
\left\{\begin{aligned}
x_{M_{2}}^{(2)}= & {\left[(m-a) \cos \theta-\left(n \pi+n t-b+\frac{r_{2} m}{\sqrt{n^{2}+m^{2}}}\right) \sin \theta\right] . } \\
& \cdot \cos \frac{t+\pi}{i_{12}}+\frac{r_{2} n}{\sqrt{n^{2}+m^{2}}} \sin \frac{t+\pi}{i_{12}} \\
y_{M_{2}}^{(2)}= & -\left[(m-a) \cos \theta-\left(n \pi+n t-b+\frac{r_{2} m}{\sqrt{n^{2}+m^{2}}}\right) \sin \theta\right] . \\
& \cdot \sin \frac{t+\pi}{i_{12}}+\frac{r_{2} n}{\sqrt{n^{2}+m^{2}}} \cos \frac{t+\pi}{i_{12}} \\
z_{M_{2}}^{(2)}= & -(m-a) \sin \theta-\left(n \pi+n t-b+\frac{r_{2} m}{\sqrt{n^{2}+m^{2}}}\right) \cos \theta
\end{aligned}\right.
$$

Comparing Eqs. (21) and (29), it can be noticed that the driving center curve only has a relationship with the driving contact curve and the driving tine radius $\left(r_{1}\right)$; comparing Eqs. (26) and (30), the driven center curve only has a relationship with the driven contact curve and the driven tine radius $\left(r_{2}\right)$. That is to say, the driving and driven center curves are irrelevant from each other and either $r_{1}$ or $r_{2}$ can be selected according to their own demanding strength.

\section{VIRTUAL SIMULATION}

The equations of the driving and driven contact curves are unique if and only if the following six parameters are given: $m, n, a, b, \theta$ and $i_{12}$. During the virtual simulation, the same pair of contact curves as in the example are used. The six parameters are selected as below: $m=5 \mathrm{~mm}, n=4 \mathrm{~mm}, a=24 \mathrm{~mm}, b=10 \mathrm{~mm}$, $\theta=120^{\circ}$ and $i_{12}=3$.

Substituting the parameters into Eqs. (21) and (26), the equations of the driving and driven contact curves as Eqs. (31) and (32) are obtained. The virtual simulation is shown in Fig. 5.

$$
\begin{gathered}
\left\{\begin{array}{l}
x_{M}^{(1)}=5 \cos t \\
y_{M}^{(1)}=5 \sin t \\
z_{M}^{(1)}=4 \pi+4 t
\end{array} \quad\left(-\pi \leq t \leq-\frac{\pi}{2}\right),\right. \\
\left\{\begin{array}{l}
x_{M}^{(2)}=\left[\frac{19}{2}-\frac{\sqrt{3}}{2}(4 \pi+4 t-10)\right] \cos \frac{t+\pi}{3} \\
y_{M}^{(2)}=-\left[\frac{19}{2}-\frac{\sqrt{3}}{2}(4 \pi+4 t-10)\right] \sin \frac{t+\pi}{3} . \\
z_{M}^{(2)}=\frac{19 \sqrt{3}}{2}+\frac{1}{2}(4 \pi+4 t-10)
\end{array} .\right.
\end{gathered}
$$

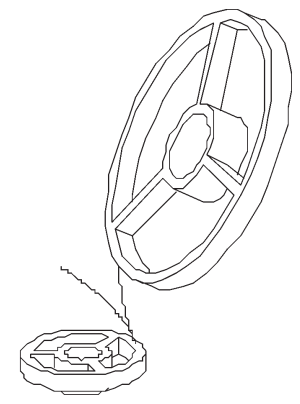

Fig. 5. Simulation of the contact curves

Substituting the parameters into Eqs. (29) and (30), the equations of the driving and driven center curves as Eqs. (33) and (34) are obtained.

$$
\left\{\begin{array}{l}
x_{M_{1}}^{(1)}=5 \cos t-\frac{4 r_{1} \sin t}{\sqrt{4^{2}+5^{2}}} \\
y_{M_{1}}^{(1)}=5 \sin t+\frac{4 r_{1} \cos t}{\sqrt{4^{2}+5^{2}}} \\
z_{M_{1}}^{(1)}=4 \pi+4 t-\frac{5 r_{1}}{\sqrt{4^{2}+5^{2}}}
\end{array}\right.
$$

$$
\left\{\begin{aligned}
x_{M_{2}}^{(2)}= & {\left[\frac{19}{2}-\frac{\sqrt{3}}{2}\left(4 \pi+4 t-10+\frac{5 r_{2}}{\sqrt{4^{2}+5^{2}}}\right)\right] } \\
& \cdot \cos \frac{t+\pi}{3}+\frac{4 r_{2}}{\sqrt{4^{2}+5^{2}}} \sin \frac{t+\pi}{3} \\
y_{M_{2}}^{(2)}= & -\left[\frac{19}{2}-\frac{\sqrt{3}}{2}\left(4 \pi+4 t-10+\frac{5 r_{2}}{\sqrt{4^{2}+5^{2}}}\right)\right] \\
& \cdot \sin \frac{t+\pi}{3}+\frac{4 r_{2}}{\sqrt{4^{2}+5^{2}}} \cos \frac{t+\pi}{3} \\
z_{M_{2}}^{(2)}= & \frac{19 \sqrt{3}}{2}+\frac{1}{2}\left(4 \pi+4 t-10+\frac{5 r_{2}}{\sqrt{4^{2}+5^{2}}}\right)
\end{aligned}\right.
$$

Once the values of $r_{1}$ and $r_{2}$ are determined, there are unique center curves corresponding with the contact curves. Therefore, the shape of the driving and driven tines can be simulated in Pro/E.

In the industry, the radii of the tines are given according to the actual need. However, to testify to the irreverence of the driving and driven tines, both $r_{1}$ and $r_{2}$ with various values are chosen and matched as pairs. To avoid interference, the radii would be assured to be in a reasonable range, which can be easily guaranteed during the simulation. 
Suppose the numbers of the driving and driven tines are $z_{1}$ and $z_{2}$, respectively. If $z_{1}=5$, according to the definition of the transmission ratio, we derive that $z_{2}=15$. As the numbers of the driving and driven tines are defined, then the shape of the SCMW is fixed, as shown in Fig. 6.
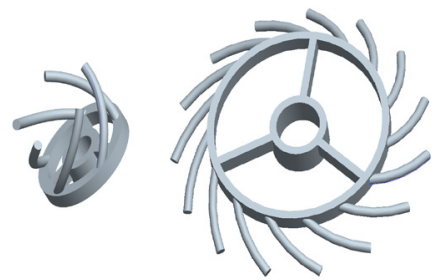

Fig. 6. Simulation of the driving and driven wheels

The meshing of the SCMW is shown as in Fig. 7. The simulation results show that the tines always mesh at the same pair of contact curves and that the value of neither $r_{1}$ nor $r_{2}$ will affect the meshing.

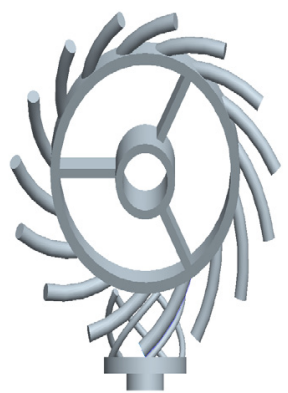

Fig. 7. Simulation of the SCMW

\section{KINEMATIC EXPERIMENT}

Using the data from the simulation above (also seen in Table 1), we manufacture some SCMW samples to do experiment to testify the irrelevance of the driving and driven wheels. The samples are produced through Selective Laser Melting (SLM) technology [11]. All the driving wheels have the same driving contact curves, as shown in Table 1, but different tine radii $\left(r_{1}\right)$, as shown in Fig. 8a; all the driven wheels have the same driven contact curves, as shown in Table 1, but different tine radii $\left(r_{2}\right)$, as shown in Fig.8 (b).

Table 1. Uniform parameters of SCMW samples

\begin{tabular}{cccccccc}
\hline$m$ & $n$ & $a$ & $b$ & $\theta$ & $i_{12}$ & $z_{1}$ & $z_{2}$ \\
\hline $5 \mathrm{~mm}$ & $4 \mathrm{~mm}$ & $24 \mathrm{~mm}$ & $10 \mathrm{~mm}$ & $120^{\circ}$ & 3 & 5 & 15 \\
\hline
\end{tabular}

The test rig made by our research group [1] to [3] is adopted to do the experiment. The experimental schematic diagram is shown in Fig. 9, and the test rig is shown in Fig. 10.

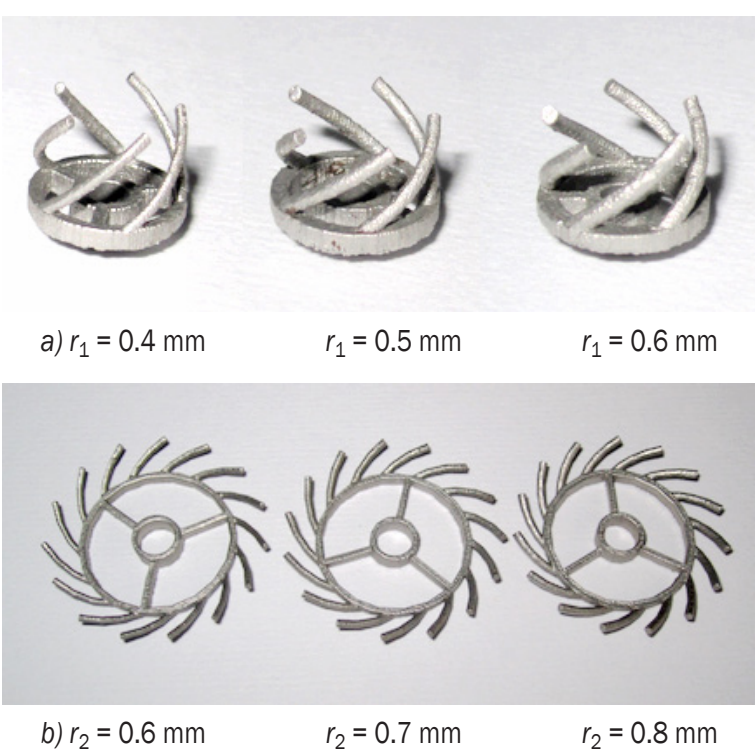

Fig. 8. SCMW samples; a) samples of driving wheels, b) samples of driven wheels

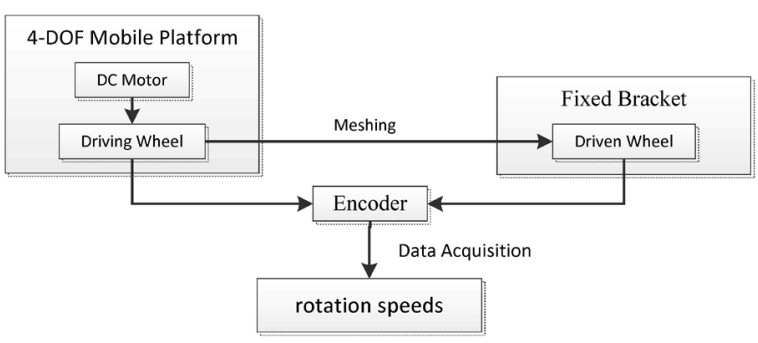

Fig. 9. Experimental schematic diagram

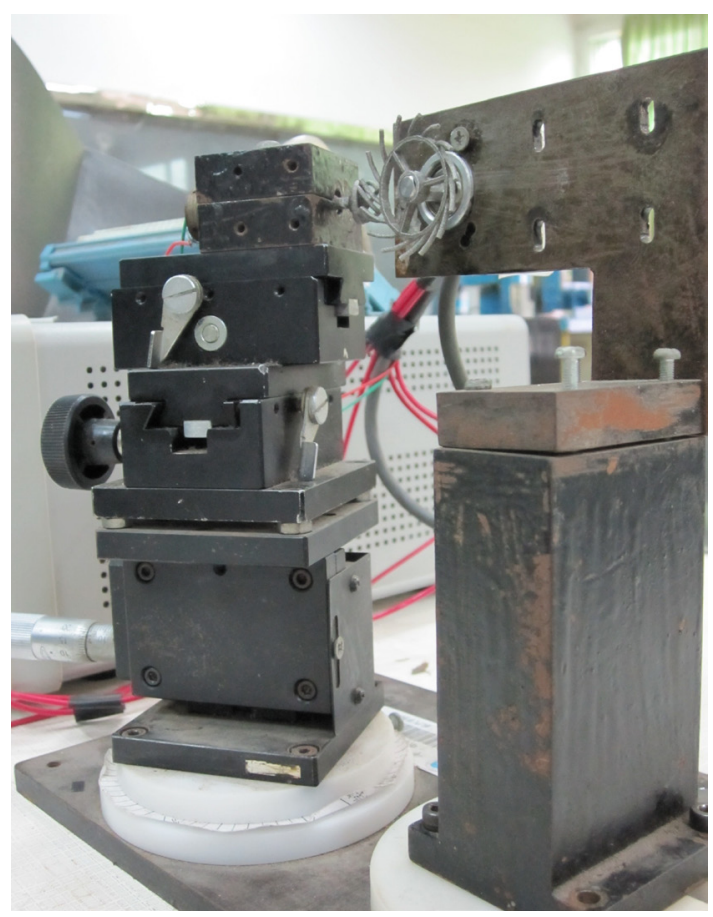

Fig. 10. Test rig 
In the experiment, to keep the pair of contact curves constant and match the driving and the driven wheels with different tine radii each time, continuous records of contact tines at different locations are captured with high quality camera. For example, as shown in Fig. 11 are continuous records of the mesh between the driving tines with $r_{1}=0.5 \mathrm{~mm}$ and the driven tines with $r_{2}=0.8 \mathrm{~mm}$.

After recording the rotation speeds of the driving and the driven wheels for a while, the corresponding average ratios are calculated. As shown in Table 2, the data in the same column indicate the meshing between the same driving wheel and different driven wheels, while the data in the same row indicate the meshing between the driving wheels and the same driven wheel.

Table 2. Average ratio of SCMW with the same pair of contact curves

\begin{tabular}{|c|c|c|c|c|}
\hline \multirow{2}{*}{$i$} & & \multicolumn{3}{|c|}{$r_{1}[\mathrm{~mm}]$} \\
\hline & & 0.4 & 0.5 & 0.6 \\
\hline \multirow{3}{*}{$r_{2}[\mathrm{~mm}]$} & 0.6 & 2.98 & 3.00 & 3.01 \\
\hline & 0.7 & 3.02 & 2.99 & 2.98 \\
\hline & 0.8 & 3.01 & 3.00 & 3.02 \\
\hline
\end{tabular}

From Table 2, the average transmission ratio measured is from 2.98 to 3.02 , while the theoretical transmission ratio is 3 . The maximum relative error is $0.6 \%$. This relative error is the combined result of manufacturing errors, assembly errors and measure errors. It can be reduced by improving manufacturing technics and experiment conditions.

It is noteworthy that the lengths of the tines do not affect the error in theory. However, as the tines are made through SLM technology, their manufacturing error accumulates as they become longer.

During the experiment, the meshing of the driving and driven wheels with unequal radii in acceptable accuracy was accomplished. From similar continuous records like Fig. 11, it has been confirmed that the tines obtained always mesh at the same pair of contact curves, so the tine radii of the driving and driven wheels are irrelevant from each other. It can be concluded that the method presented is reliable.

\section{CONCLUSION}

In this paper, a new method based on the contact curves is proposed to design the SCMWs with unequal tine radii. It is illustrated with a design example in detail, and testified with both simulation and experiment. In comparison with existing design methods, the method presented has two obvious advantages as below:

1) The radii of the driving and driven tine do not affect the meshing process, and the radii of the driving and driven tines can be designed independently according to their working demands.

2) It is the theoretical foundation of the tine shape optimization based on the strength condition.

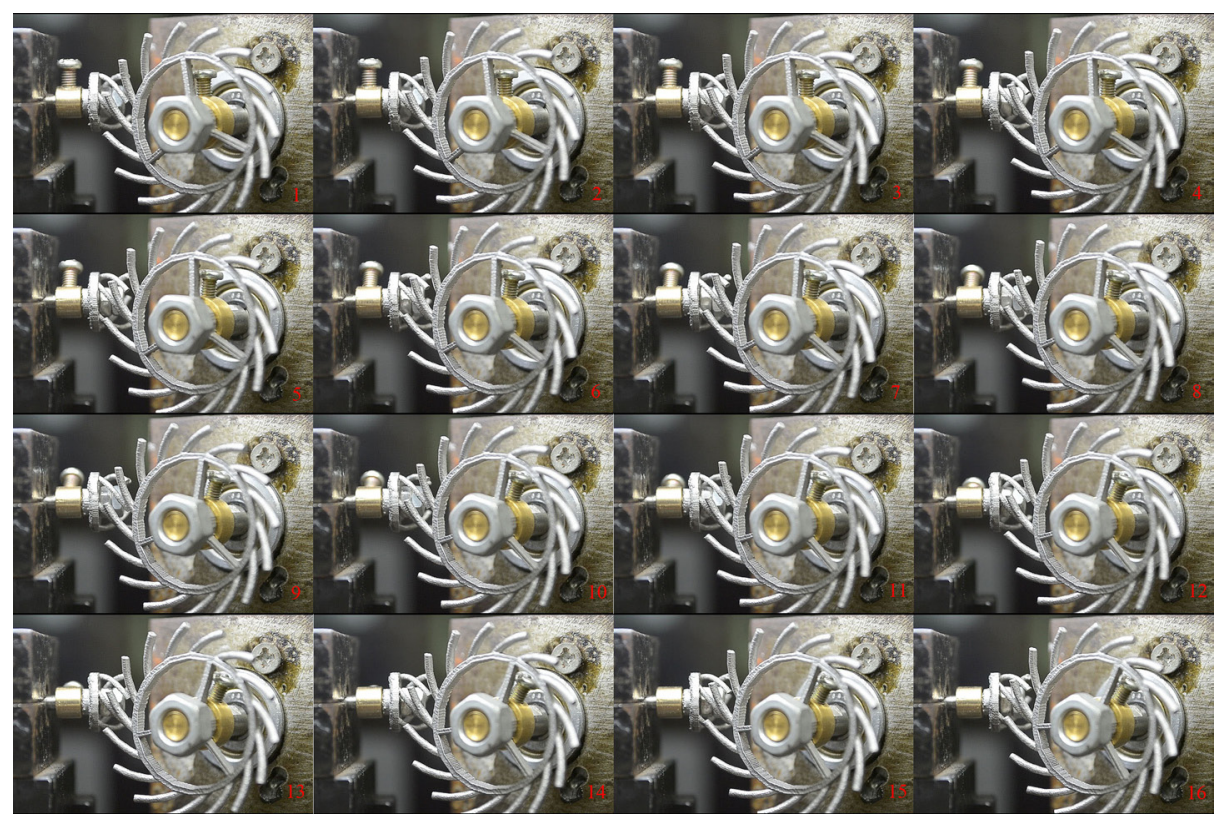

Fig. 11. Continuous records of contact tines at different locations 
This method possesses several prospects to conduct further study. With this method, even the SCMW with variable radii and invariable stress can be designed. To identify the optimized radii, the relationship between the stresses and the radii is in progress. The shape selection rule would be established afterward.

\section{ACKNOWLEDGMENT}

It is our pleasure to thank anonymous reviewers and editors for their valuable comments and suggestions. We thank the National Natural Science Foundation of China (No. 51175180) for the support of the research presented in this paper.

\section{NOMENCLATURE}

$\begin{array}{ll}r_{1} & \text { Radius of driving tine } \\ r_{2} & \text { Radius of driven tine } \\ \varphi_{1} & \text { Rotation angle of driving wheel } \\ \varphi_{2} & \text { Rotation angle of driven wheel } \\ \varpi_{1} & \text { Angular velocity of driving wheel } \\ \varpi_{2} & \text { Angular velocity of driven wheel } \\ v_{1} & \text { Velocity of driving tine } \\ \boldsymbol{v}_{2} & \text { Velocity of driven tine } \\ \boldsymbol{v}_{12} & \text { Relative velocity } \\ i_{12} & \text { Transmission ratio } \\ \theta & \text { Included angle between angular } \\ & \text { velocity vectors } \\ \mathrm{a} & \text { Distance from point } o_{p} \text { to axis } z \\ \mathrm{~b} & \text { Distance from point } o_{p} \text { to axis } z \\ M_{o 1} & \text { Transformation matrix from } o_{1}-x_{1} y_{1} z_{1} \\ & \text { to } o-x y z \\ M_{21} & \text { Transformation matrix from } \\ \mathrm{t} & o_{1}-x_{1} y_{1} z_{1} \text { to } o_{2}-x_{2} y_{2} z_{2} \\ \beta & \text { Scope parameter of helix curve } \\ \gamma & \text { Unit normal vector of driving curve } \\ \mathrm{M} & \text { Unit binormal vector of driving curve } \\ M^{(1)} & \text { Meshing point } \\ M^{(2)} & \text { Point at driving contact curve } \\ M_{1} & \text { Point at driven contact curve } \\ M_{2} & \text { Point at driving center curve } \\ \overrightarrow{M M_{1}} & \text { Point at driven center curve } \\ \overrightarrow{M M_{2}} & \text { Contact vector from } M \text { to } M_{1} \\ \mathrm{~m} & \text { Contact vector from } M \text { to } M_{2} \\ \mathrm{n} & \text { Helix radius of driving curve } \\ p & \text { Pitch parameter of driving curve } \\ & \text { Pitch of driving curve } \\ & \end{array}$

$\begin{array}{ll}z_{1} & \text { Number of driving tines } \\ z_{2} & \text { Number of driven tines } \\ \text { (superscript) } & \text { Corresponding coordinate }\end{array}$

\section{REFERENCES}

[1] Chen, Y.Z., Xing, G.Q., Peng, X.F. (2007). The space curve mesh equation and its kinematics experiment. $12^{\text {th }}$ IFToMM World Congress, Besançon.

[2] Chen, Y.Z., Xiang X.Y., Luo, L. (2009). A corrected equation of space curve meshing. Mechanism and Machine Theory, vol. 44, no. 7, p. 1348-1359, DOI:10.1016/j.mechmachtheory.2008.11.001.

[3] Chen, Y.Z., Chen, Z., Fu, X.Y. (2011). Design parameters for spatial helix gearing mechanism. Applied Mechanics and Materials, vol.121-126, p. 3215.

[4] Litvin, F.L. (2008). Gear Geometry and Applied Theory. Shanghai Science and Technology Publishers, Shanghai. (in Chinese)

[5] Bergseth, E. Björklund, S. (2010). Logarithmical crowning for spur gears. Strojniški vestnik - Journal of Mechanical Engineering, vol. 56, no. 4, p. 239-244.

[6] Fetvaci, C. (2010). Generation simulation of involute spur gears machined by pinion-type shaper cutters. Strojniški vestnik - Journal of Mechanical Engineering, vol. 56 , no. 10 , p. 644-652.

[7] Staniek, R. (2011). Shaping of face toothing in flat spiroid gears. Strojniški vestnik - Journal of Mechanical Engineering, vol. 57, no. 1, p. 47-54, DOI:10.5545/svjme.2010.093.

[8] Puccio, F.D., Gabiccini, M., Guiggiani, M. (2006). Generation and curvature analysis of conjugate surfaces via a new approach. Mechanism and Machine Theory, vol. 41, no. 4, p. 382-404, DOI:10.1016/j. mechmachtheory.2005.07.008.

[9] Chen, Y.Z., Luo, L., Hu, Q. (2009). The contact ratio of a space-curve meshing-wheel. Journal of Mechanical Design, vol. 131, no. 7, p. 074501, DOI:10.1115/1.3116343.

[10] Chen, Y.Z., Hu, Q., Su, L. (2010). Design criterion for the space-curve meshing-wheel transmission mechanism based on the deformation of tines. Journal of Mechanical Design, vol. 132, no. 5, p. 054502, DOI:10.1115/1.4001535.

[11] Chen, Y.Z., Sun, L.H, Wang, D., Yang, Y.Q., Ding, J. (2010). Investigation into the process of selective laser melting rapid prototyping manufacturing for spacecurve-meshing-wheel. Advanced Material Research, vol. 135, p. 122-127, DOI:10.4028/www.scientific.net/ AMR.135.122.

[12] Chen, Y.Z., Chen, Z., Ding, J. (2011). Space Curve Mesh Driving Pair and Polyhedral Space Curve Mesh Transmission, PCT/CN2010/078294.

[13] Li, G.Y. (2007). Spatial Geometry Modeling and Its Application in Engineering, Higher Education Press, Beijing. (in Chinese) 